\title{
CAR-DSDV: CONTEXT AWARE ROUTING IN DELAY TOLERANT NETWORK (DTN)
}

\author{
Pankaj Kumar Gautam ${ }^{1}$, Pranshu Mittal ${ }^{2}$, Sapna Chaudhary ${ }^{3}$, Rahul Johari ${ }^{4 *}$ and \\ Arsala Saleem ${ }^{5}$ \\ 1,2,3,4,5 University School of Information, Communication and Technology, GGSIP \\ University, Sector-16C, Dwarka, India \\ ${ }^{1}$ pankaj.usict.017164@ipu.ac.in, ${ }^{2}$ pranshu2009@gmail.com, \\ ${ }^{3}$ spnchaudhary29@gmail.com, ${ }^{4}$ rahul@ipu.ac.in, ${ }^{5}$ arslawar31@gmail.com
}

\begin{abstract}
DTN is a system of smaller network and is an overlay on the top of the internet that supports inter-operability by accommodating long disruption/delay between and within SHIP(Sparse, Hybrid, Intermittent and Partitioned) networks. During the literature survey, it was found that research and implementation is being carried out in different areas of DTN like Routing, Quality of Service, Buffer Space Management, Node Energy Optimization, and Security. In the current research work, it is described in detail how local context information related to network nodes can be exploited to develop a routing scheme that offers similar message delivery statistics as other schemes and drastically reduces network traffic at the cost of a little higher latency. Such a scheme is of considerable use to network providers who wish to extend their services to sparsely populated areas like mountains, deserts, etc.
\end{abstract}

Keywords - DTN, SHIP networks, Internet of Things (IoT) environment, context calculation, node co-location

\section{INTRODUCTION}

DTN is a general purpose overlay network on the top of internet that takes into consideration the communication needs of wireless military network, outer-space network and various such networks. It is a network of smaller networks that support interoperability by accommodating long delays and disruptions between the networks.Compared to traditional routing, the routing of messages is quite different in this kind of environment. Thus, the need for finding new routing protocols has been created which can take efficiently into account the different nature of these networks.

Delay Tolerant Network overcome the problems related with prolong delays,irregular connectivity, varying delay, unsymmetrical data rates and inflated error rates by using store-and-forward message switching technique as depicted in Section 3.

\subsection{ARCHITECTURE}

The DTN architecture embraces the message switching for occasionally connected networks by a protocol called bundle protocol where message aggregates are called "bundles" and "bundle forwarders" are the routers that handle those bundles. The agent of bundle-protocol accumulate and then forwards the complete bundle between the nodes and therefore DTN implements store-and-forward message switching by this protocol [1].

Received: September 21, 2019

Reviewed: November 19, 2019

Accepted: December 2, 2019

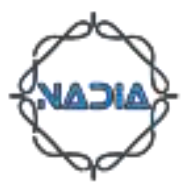


To enhance end to end reliability in DTNs, the bundle protocol implements custodial transfer. Once an intermittent node receives a bundle message it can take custody of that message so that possible re-transmissions can be done from the intermittent node itself instead of re-transmitting it all the way back from the source node [2].

\subsection{NODE CONNECTIONS}

The different types of connection/contacts that can occur between nodes of a DTN are broadly classified as follows [20]:

A. Persistent: In this the connection is always available and there is no need of connection-initiation action in order to incorporate a persistent contact. Cable Modem connection an 'always-on' connection of internet would be a representative of this class.

B. On-demand: This function as persistent contacts till termination but require action to instantiate. The example of this is a dial-up connection.

C. Scheduled: To establish contact for a specific duration, at a specific time a scheduled contact act as an agreement. Low-earth orbiting satellite linking is an example of scheduled contact. By using the satellite's scheme of capacities, latency and view times a list of node's contacts with a satellite can be constructed.

D. Opportunistic: They present themselves unexpectedly and are not scheduled. For example, an unplanned aircraft flying beckoning and overhead, publicizing its availability for the purpose of communication would present an opportunistic contact. A Bluetooth or infrared communication link between a kiosk and PDA in an airport concourse is another kind of opportunistic contact.

E. Predicted: Based on no fixed schedule, but rather are predictions of likely contact times and duration based on a history of prior observed contacts or some other information.

\subsection{APPLICATION}

Some of the possible scenarios for application of DTNs are stated below:

A. Space Agencies: Interplanetary communication, International Space Station(ISS) communication (in research filed), space-trash monitoring.

B. Public Service \& Safety: Search and rescue communication, security and disaster communication, global airport-traffic control, humanitarian relief monitoring, smart transportation networks.

C. Environment Monitoring: Relocation of animal, soil stability and properties, atmospheric, oceanographic conditions, and seismological event.

D. Scientific \& Engineering Research: Subject-matter experts of a network, academic research by both faculty \& students.

E. Military: Mobile ad-hoc networks for wireless monitoring \& communication, search \& rescue, UAV communication and control.

F. Undersea Communication: Undersea vehicles, oil and mining, submarines and under-surface sensors which are unnamed.

\section{LITERATURE SURVEY}

Reference [3] focuses on the improved VDTN (Vehicle Delay Tolerant Network) routing performance. Active Area-based routing approach consists of two levels based on the count of operating sub-areas frequently visited by the vehicle and two frequently encountered vehicles encountering in their active sub-areas. The first level consists of a spreading algorithm which distributes duplicate of the destination vehicle packet to each operating sub-area. In the second level, the high meeting frequency vehicle with respect to the destination vehicle gets the forwarded packet from each packet carrier. The result 
of the simulation is that AAR produces less delay and greater success rate compared to other routing algorithms in VDTNs.

Reference [4] focuses on reviewing the literature of existing Delay Tolerant Network protocols by using different metrics like MDR(Message Delivery Ratio) and average delay.

Reference [5] focuses on the protocol for routing that dispense reduced delays and better performance and therefore a protocol which supply data packet with less delay and less amount of time.

Reference [6] focuses on the evaluation tools for testing the routing schemes proposed for providing communication services in DTNs. It also focuses on the trend of the emulation which support large scale mobility trace. TUNIE is proposed for DTN protocol evaluation which is a large scale emulation. The proposed TUNIE provide realistic DTN environment, less time-consuming and is less costly. It also evaluate the system performance accurately. The result of system implementation and system prototype is for evaluating the DTN protocol performance. TUNIE is a flexible platform.

Reference [7] focuses on a source-based routing technique. To represent various characteristics like maximum buffer space, buffer space available and community information of node's CONCOR is defined that define these characteristics. CONCOR differ from other in a way that if a node comes in contact with station only then it becomes a member of a group.

Reference [8] focuses on the new routing algorithm for efficient message delivery in DTN. Dynamic common structure is formed by the nodes with common point of interest and the utility function is formulated by identifying the node's attribute in order to determine the delivery capability of a message. A node with more delivery probability then the node itself is selected for message forwarding. Metrics like MDR (Message Delivery Ratio), average delay are used for comparing the new routing algorithm with DSG and CAR.

Reference [9] focuses on different routing scheme in which a utility function is used to calculate whether to send a message to a scheduled contact or an opportunistic contact and thus the algorithm has improved message traffic ratio and message delivery ratio.

Reference [10] focuses on some modifications in the routing metric calculation done in existing PROPHET routing scheme which reduce certain issues and improved protocol performance and hence the updated version of the protocol is called PROPHETv2.

Reference [11] focuses on the prediction of user future movement in an opportunistic networks. To convey the content to interested users a context- aware and social - aware context sharing is used. Also, the existing architecture is integrated with the context sharing service and the middle-ware. The proposed context sharing work is validated on large scale and on a small scale and the benefits of it is investigated by the simulation done. Thus the result of this paper is that in opportunistic networks the performance and efficiency of services and protocols is improved.

Reference [12] focuses on designing of a routing scheme called COMFA based on the regular pattern of movement of the members of community. The routing algorithm exploit this pattern and then construct a routing table and a decentralized solution to maximize the message delivery probability and on comparing with other routing schemes it has better message delivery while reducing the overhead of message significantly.

Reference [13] focuses on designing a routing scheme where a series of groups on the basis of node's regular contacts patterns called social groups are formed, these social groups uses probabilistic routing scheme to identify consistently contacting groups with base station and thus route accordingly and therefore can be dynamically implemented by maintaining a high delivery ratio.

Reference [14] proposes an algorithm called BUBBLE which is social based forwarding algorithm. Also this paper focuses on improving the understanding of the mobility of human in a social structure and then using those structures to design such 
forwarding algorithms for PSNs thus showing how BUBBLE algorithm can be implemented in a distributed way.

Reference [15] focuses on a routing framework called SocialCast that utilize predictions based on the social movement patterns among the communities in order to find out the best information carrier for publish-subscribe.

Reference [16] focuses on the routing scheme which has been suggested to deal with intermittently connected networks therefore the flooding-based routing scheme is used as it has high probability of delivery but wastage of energy is more hence a routing scheme is proposed called Spray and Wait in which multiple copies are sprayed into the network and then waits for one of the node that meets the destination.

Reference [17] focuses on the routing scheme which is a variant of existing routing scheme named epidemic routing used for intermittent networks called PROPHET, a Probabilistic Routing Protocol which is used for sparse mesh networks.

Reference [18] focuses on the message delivery techniques in cases where there is no path from source to destination, for this an author introduce an Epidemic routing where pair-wise message exchange takes place among the mobile host, the goal of this routing technique is to increase the latency of a message and to decrease the total count of resources consumed during delivery of a message.

\section{METHODOLOGY ADOPTED}

Routing is one of the major components of the DTN protocol stack, others being QoS, Security, Buffer Space Management etc. Most of the current approaches targeting routing in DTNs are either flooding based (e.g., epidemic routing, spray and wait) or probabilistic (PRoPHET). These routing schemes can be further classified as follows:

A. Replication based routing protocols are those that replicate message. These protocols have high message delivery rates with lower transmission times and high robustness. But these advantages come at the cost of high network traffic.

B. Forwarding based routing protocols are those that don't replicate message packet. These are similar to protocols used in traditional TCP/IP network stacks. They significantly cut down on network traffic consequently offering a higher QoS but they are usually slower and less robust than replication based protocols.

None of the popular approaches currently exploit the context-based information possessed by network nodes (such as co-location history). A context-based routing protocol is described in the Section 3 and Section 4 that work on the forwarding based approach.

Current research on context-based routing considers two important context parameters, namely change in the degree of connectivity and current co-location.

While co-location data works well for evaluating context over short distances, it is of limited use when spread over large transmission distances, a common occurrence in a lot of DTN scenarios. Hence, a scene co-location based context parameter is augmented here along with other context information.

In theory, scene recognition works well when relevant context information is to be established over large distances.

The best case scenario that applies to this approach is a small-world network [19] spread over large geographical distances. A small-world network in which neighbors of any given node are neighbors of each other and by only a few number of steps or hops many nodes can reach from every other node and it is a kind of mathematical graph. This small-world network is a network where $L$ (hop distance) between two nodes which are randomly chosen proportionally grows to the logarithm of $N$ (number of nodes) in the network, i.e;

$$
L \alpha \log (N)
$$


The proposed algorithm uses synchronous routing like DSDV (Destination-Sequenced Distance Vector Routing) to relay messages between connected nodes.

If the destination exists in a different network cloud due to a network partition, the asynchronous routing based on context is used to select the next best-connected node that has the best chance of connecting with the destination in future. This transfer to the best node is then done by the underlying synchronous protocol.

A. Dest(Index): It denotes the destination. This field is used to index the table.

B. Distance: It indicates the distance of current node from the destination. This field is used for synchronous routing.

C. NextHop: It denotes the best next hop for synchronous routing in accordance with Destination-Sequenced Distance Vector (DSDV) Routing.

D. DeliveryProb: It denotes delivery probability and is used for asynchronous routing.It is calculated by using the Eq. 4.

E. BestHop: It denotes the next best hop having the highest delivery probability.

F. SeqNo: It denotes sequence number and is used to keep track of routing table updates for implementing DSDV.

Table I. Routing Table Format

\begin{tabular}{|l|l|l|l|l|l|}
\hline Dest(Index) & Distance & NextHop & DeliveryProb & BestHop & SeqNo \\
\hline 1 & 4 & 2 & 0.7 & 3 & 1 \\
\hline$\cdot$ &. &. &. &. &. \\
$\cdot$ & $\cdot$ & $\cdot$ & $\cdot$ & $\cdot$ &. \\
. &. &. &. &. &. \\
\hline 20 & 3 & 7 & 0.5 & 3 & 20 \\
\hline
\end{tabular}

\subsection{MATHEMATICAL MODEL}

Symbols used:

$n$ : number of nodes

$n_{i}: i$ - th host node in the netw ork

$d_{i}: i$ - th destinat - ion node in netw ork

$U(X)$ : utility function fo CAR

$U(x)$ :sum of all utility functions

$t$ :instantane- ous time

$T$ :time perid of simulation

$a_{i}$ :adaptive weight

$w_{i}$ :statically set weight

Context is defined as a set of attributes $\left\{X_{1}, X_{2}\right\}$ where,

$X_{1}=$ changein degree of connectivity,

$X_{2}=$ coloc ation measuredby DVR table exchange, and

$N=$ set of nodes in the network

Based on these attributes, their utility functions are computed. The utility functions for node $n_{i} \in N$ are defined as follows: 
$U_{i}\left(X_{1}\right)(t)=\frac{|n(t-T) \cup n(t)|-|n(t-T) \cap n(t)|}{|n(t-T) \cup n(t)|}$

where total number of nodes is $N, n(t)$ is the number of hosts co-located with other nodes at time $t$ (the instantaneous time from the taken simulation time period $T$ ).

$U_{k, i}\left(X_{2}\right)(t)=\left\{\begin{array}{l}1, \text { if the node } k \text { is coloc ated with node } i \text { at time } t \\ 0, \text { otherwise }\end{array}\right.$

These utility functions are weighed and composed to generate an overall delivery probability of a host $n_{i} \in N$ for a destination $d_{i} \in N$.

Hence the delivery probability is,

$f\left(U\left(x_{i}\right)\right)=\sum_{i=1}^{2} a_{i} \cdot w_{i} \cdot U\left(x_{i}\right)$

where $a_{i}$ are adaptive weights whose value depends on the current context, $w_{i}$ are statically set weights based on scenario and $U\left(x_{i}\right)$ is the sum of both the utility functions defined in Eq. 2-3 for the $x$-ith node.

In general,

$a_{i}=a_{i}($ range $) \cdot a_{i}($ predictability $) \cdot a_{i}($ availability $)$

Since prediction of context values is not used, therefore $a_{i}$ (predictability) is not taken into account.

Hence,

$$
\begin{aligned}
a_{i}(\text { availability }) & =1 \text { for } i \in\{1,2\} \\
a_{1}(\text { range }) & =\log (x) \text { for } x \in(1,10) \\
a_{2}(\text { range }) & =1
\end{aligned}
$$




\subsection{FLOW CHARTS}

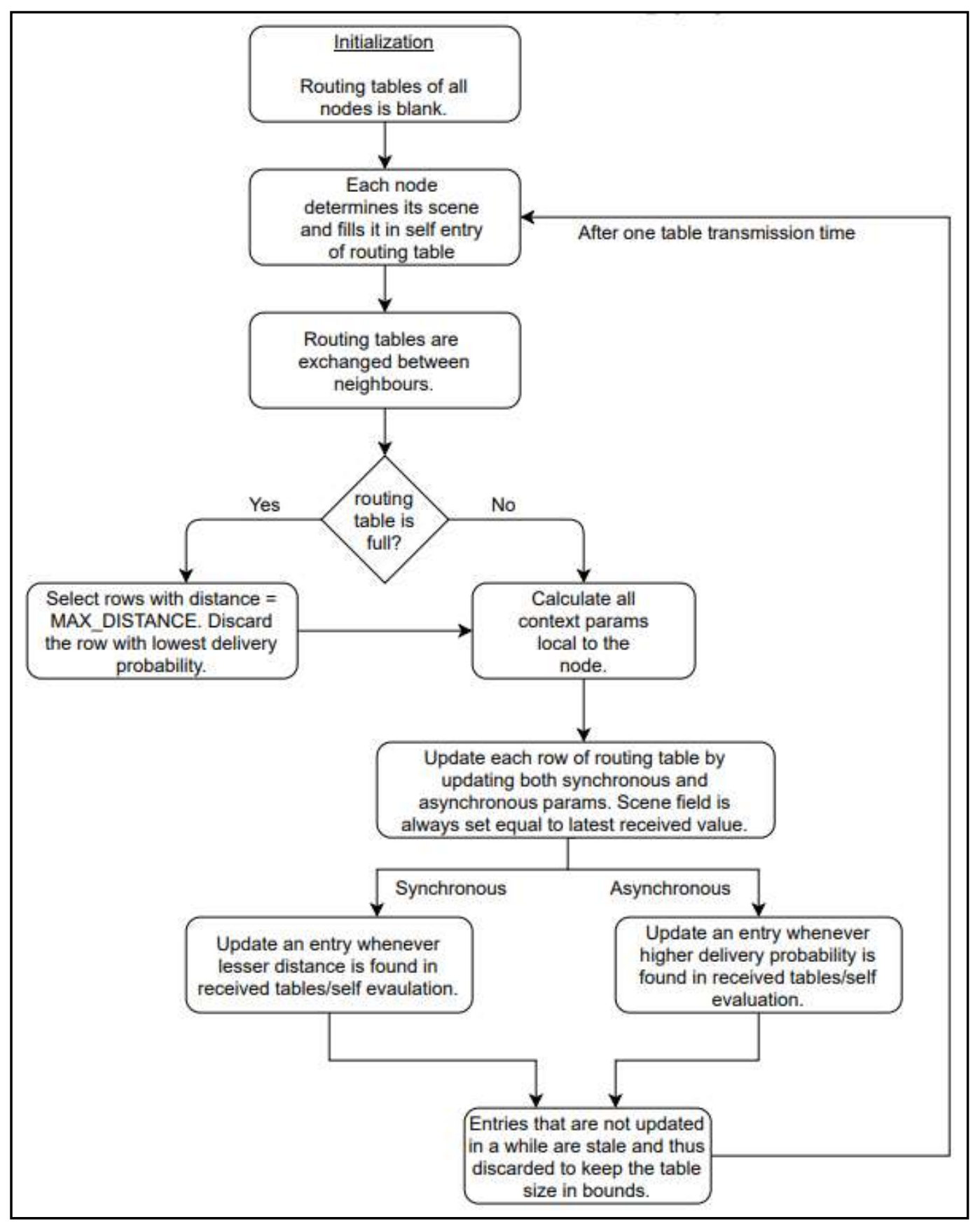

Fig. 1 Updating routing table for CAR 


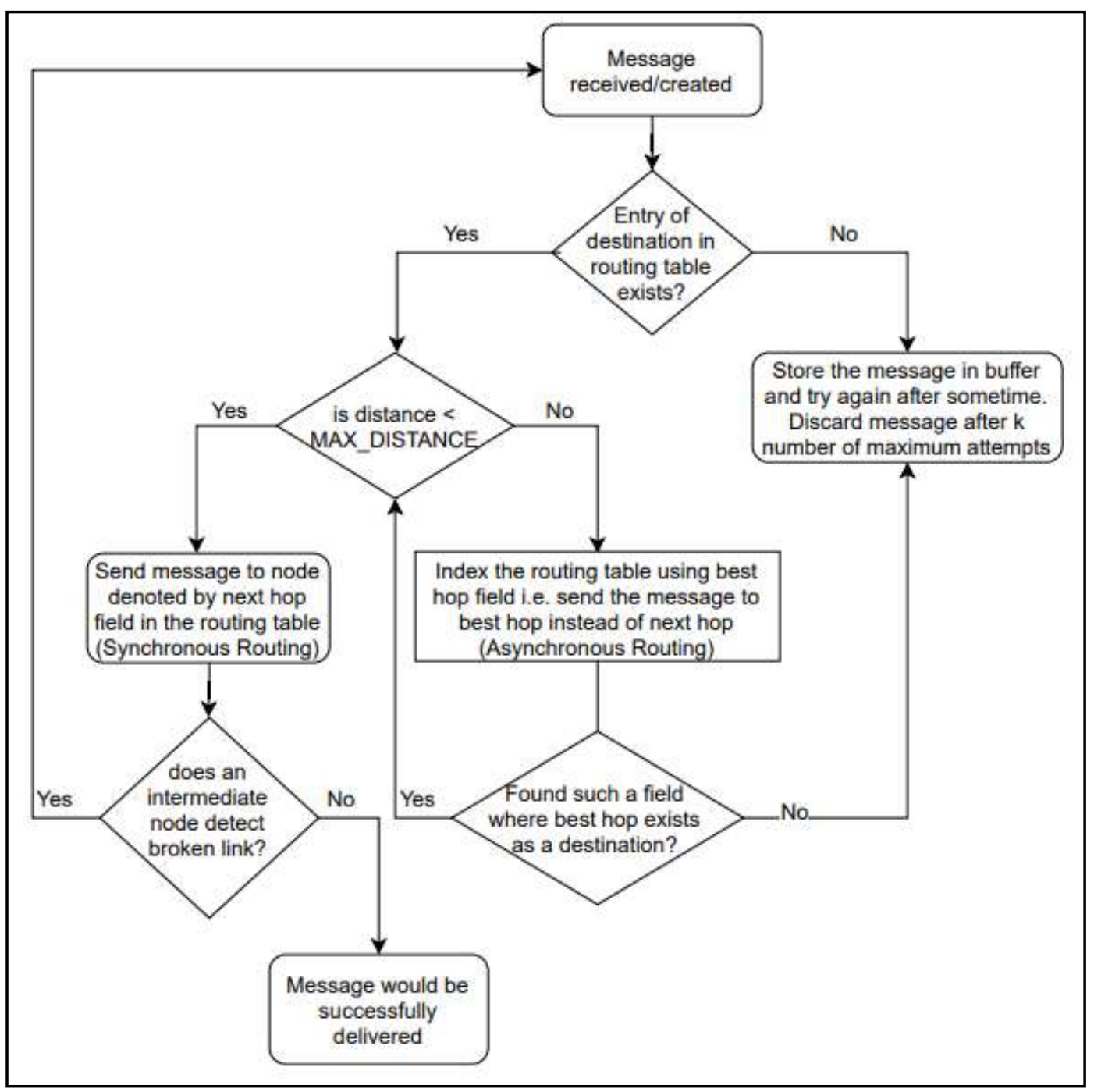

Fig. 2 Message forwarding in DTN nodes

\section{SIMULATION}

The proposed routing algorithm is simulated on ONE (Opportunistic Network Environment) simulator. Java language is used for programming in ONE simulator. In terms of software specifications for simulation, Linux OS(Ubuntu 18.04.2), Eclipse IDE 2018-09 \& Java Development Kit 1.8 have been used.

\subsection{SIMULATION PARAMETERS}

In current research work, an appropriate scenario has been chosen to simulate proposed routing scheme for message transmitting. Default settings (provided by ONE simulator) have been modified according to the proposed context-aware routing scheme. The main focus is on evaluating the performance using three metrics, Delivery Ratio, Traffic, and Latency. The implementations run with the following settings (see Table II). The mobile nodes are grouped into a single group(Pedestrian) and move in a $4500 \times 3400 \mathrm{~m}^{2}$ area which is a default scenario in ONE simulator. The pedestrian group follows "ShortestPathMapBasedMovement" movement model. 
Table II. Parameters Used For Simulation

\begin{tabular}{|l|l|l|}
\hline Attribute & Parameter & Value \\
\hline Scene & Simulated City & Helsinki city \\
\cline { 2 - 3 } & Simulation Time $(\mathrm{min})$ & $\{15,30,60,90,120\}$ \\
\cline { 2 - 3 } & Simulation Area $\left(\mathrm{m}^{2}\right)$ & $4500 \times 3400$ \\
\hline \multirow{5}{*}{ Messages } & Interval(sec) & $25-35$ \\
\cline { 2 - 3 } & Size(MB) & $0.5-1$ \\
\cline { 2 - 3 } & Message TTL(min) & 5 \\
\hline \multirow{5}{*}{ Nodes } & Movement Model & ShortestPathMapBased \\
\cline { 2 - 3 } & Transmission Interface & High-speed internet \\
\cline { 2 - 3 } & Moving Speed $(\mathrm{m} / \mathrm{s})$ & $0.5-1.5$ \\
\cline { 2 - 3 } & Group & Pedestrian \\
\cline { 2 - 3 } & Number of nodes & 20 \\
\hline Transmission & Speed(MB/s) & 10 \\
\cline { 2 - 3 } & Range(m) & 1000 \\
\hline
\end{tabular}

\subsection{EVALUATION PARAMETERS/METRICS}

The proposed work has considered three matrices for the evaluation of ContextAware routing with respect to other routing schemes like Epidemic, Prophet and MaxProp.

The three matrices are as follows:

- Message delivery ratio: It refers to the ratio of the total message delivered in a network to the total messages created into it.

- Latency: It refers to the time taken in seconds for a message to reach from source to destination.

- Message Traffic: It refers to the normalized difference of the total count of delivered messages to the destination and total count of intermediate messages relayed.

In general, if $m_{A}$ is the total count of messages delivered and $M_{A}$ is the total messages for the time period $T$ (simulation time) then,

$$
M D R^{T}=\frac{m_{A}^{T}}{M_{A}^{T}}
$$

Also, if $m_{r e l}$ is the total count of messages relayed, then Message Traffic(MT) for the time period $T$ is defined as,

$$
M T^{T}=\frac{m_{r e l}^{T}-m_{A}^{T}}{m_{A}^{T}}
$$

\section{RESULTS}

The proposed work has a series of outputs following the successful simulation using ONE simulator. Performance comparison of this protocol with the other three protocols (viz; Epidemic, Prophet, and MaxProp) is shown in Fig. 3-5. A discussion is made on the results in detail in this section.

In order to observe how the protocols (viz; Context-Aware, Epidemic, Prophet, MaxProp) react to the changes based on different simulation times, simulation time is varied from 15 to 120 minutes (see Table II), taking other simulation parameters as default provided by ONE simulator.

Fig. 3-5 summarizes all fundamental metrics of simulation output in varying simulation time(sec). The result of context-aware routing with Epidemic (Fig. 3), Prophet (Fig. 4), and MaxProp (Fig. 5). Message Traffic in CAR reduces significantly with respect to other 
routing protocols taken for comparison in each simulation period. With the increasing simulation time, other routing protocols get higher message traffic than CAR.

Also, CAR performs better than Epidemic (Fig. 3) on message delivery ratio. But other two traditional routing protocols(Prophet, Maxprop) have slightly high MDR.

Proposing context parameters to make CAR protocol more effective. It's clear to see that this routing protocol delivered better performance on message delivery ratio and message traffic compared with others, as shown in Fig 3-5. It benefits from intelligently relaying messages based on local context. Moreover, the proposed routing scheme uses synchronous DSDV routing whenever possible to send the message via the shortest available path. Lower message traffic with higher delivery rates is a testimony to the effectiveness of this routing scheme.

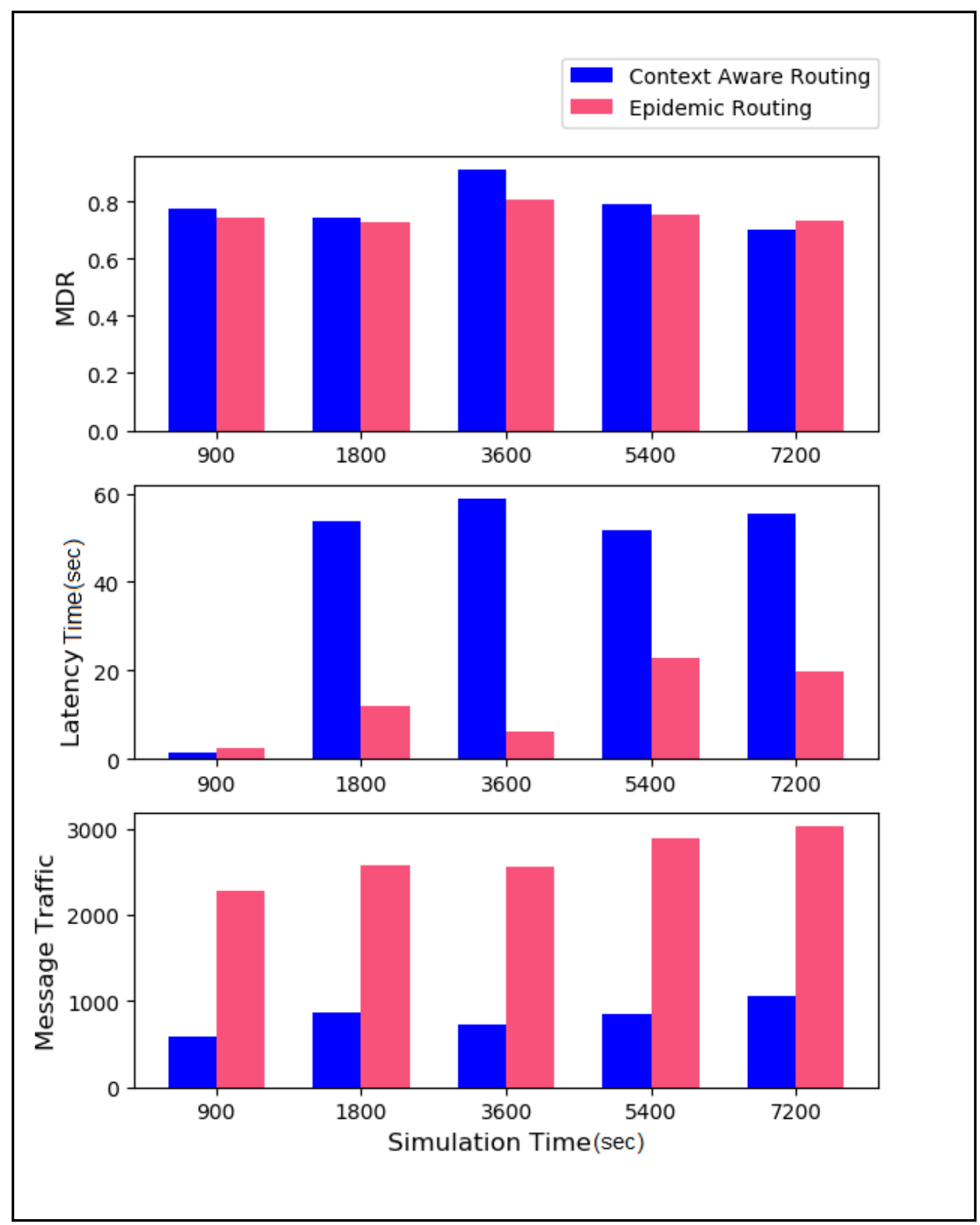

Fig. 3 Comparison between CAR \& Epidemic Routing on the basis of MDR, Latency and Message traffic, varying simulation time 


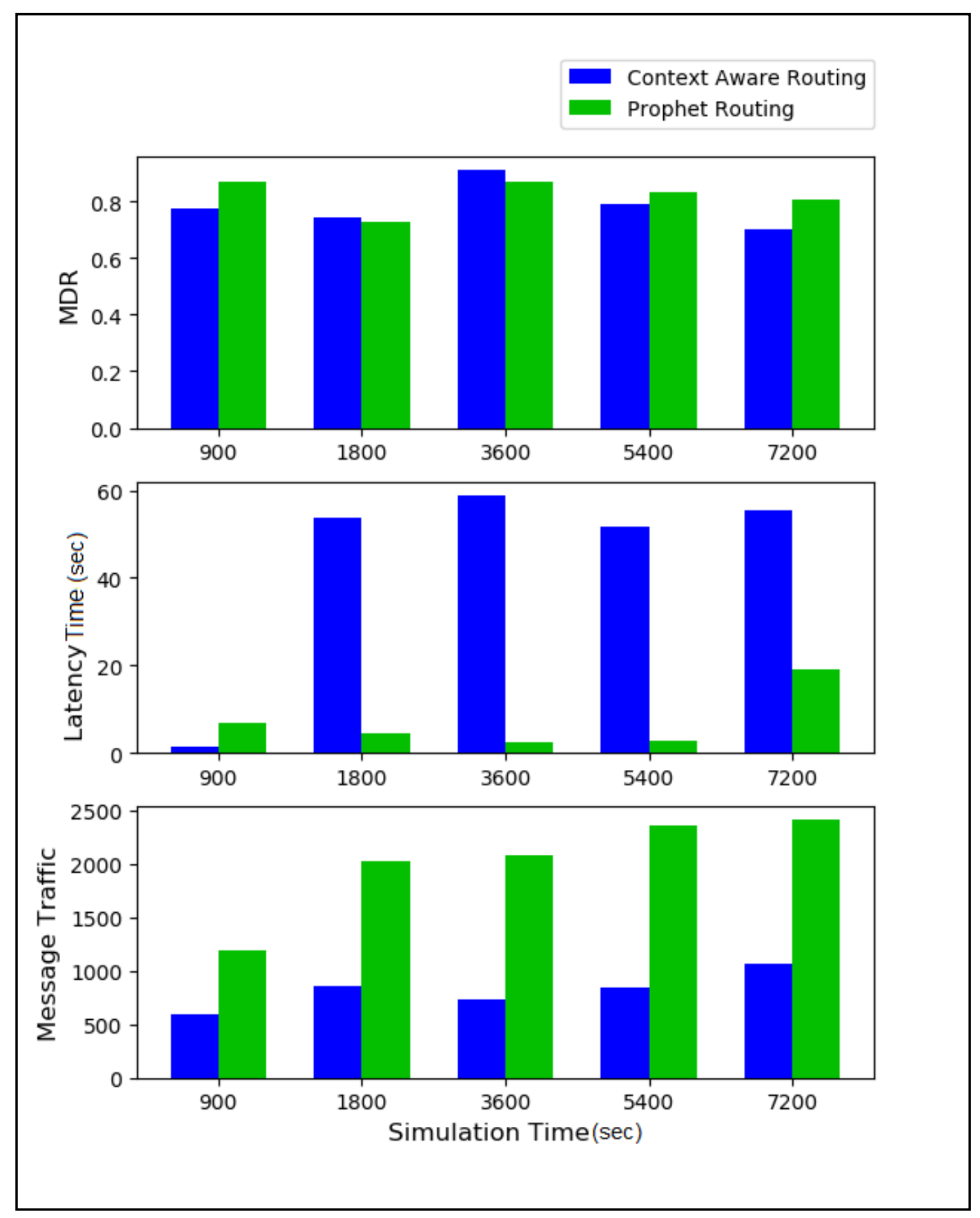

Fig. 4 Comparison between CAR \& Prophet Routing on the basis of MDR, Latency and Message traffic, varying simulation time 


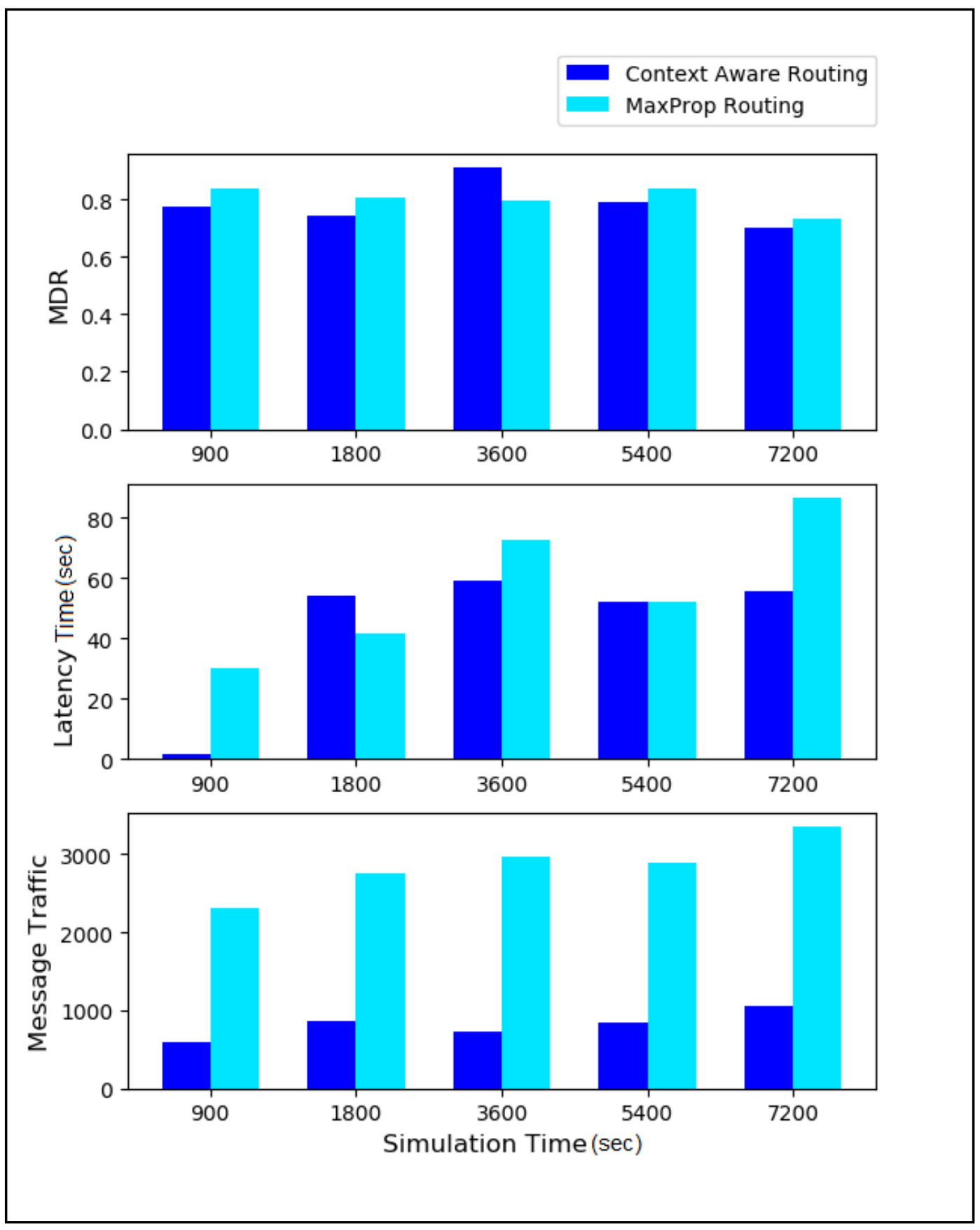

Fig. 5 Comparison between CAR \& MaxProp Routing on the basis of MDR, Latency and Message traffic, varying simulation time

\section{CONCLUSION AND FUTURE WORK}

In simulation CAR performs better than both the Epidemic and Prophet Routing Schemes on the basis of message delivery ratio and message traffic ratio.

In this paper scene/surrounding is introduced as a new context information for node. Though, due to constraints in execution time caused by the remote computation of scene information of simulation nodes, it was dropped.

In future work, it is proposed to add scene as another context attribute by using dedicated Neural Processing Units for the local computation and evaluation of scene information. 


\section{ACKNOWLEDGMENTS}

We would like to extend our sincere thanks to the faculty of GGSIP University for supporting the research project.

\section{REFERENCES}

[1] Fall, K. and Farrell, S., 2008. DTN: an architectural retrospective. IEEE Journal on Selected areas in communications, 26(5), pp.828-836.

[2] Musolesi, M. and Mascolo, C., 2009. CAR: Context-aware adaptive routing for delay-tolerant mobile networks. IEEE Transactions on Mobile Computing, 8(2), pp.246-260.

[3] Wu, B., Shen, H. and Chen, K., 2018. Exploiting active subareas for multi-copy routing in VDTNs. IEEE Transactions on Vehicular Technology, 67(5), pp.4374-4388.

[4] Singh, S., Johari, R. and Kaur, M., 2016, March. ART: Analysis of routing techniques in Delay Tolerant Network. In 2016 International Conference on Electrical, Electronics, and Optimization Techniques (ICEEOT) (pp. 2537-2542). IEEE.

[5] Johari, R. and Dhama, S., 2016. Routing protocols in delay tolerant networks: application-oriented survey. In Wireless Communications, Networking and Applications (pp. 1255-1267). Springer, New Delhi.

[6] Li, Y., Hui, P., Jin, D. and Chen, S., 2015. Delay-tolerant network protocol testing and evaluation. IEEE Communications Magazine, 53(1), pp.258-266..

[7] Johari, R., Gupta, N. and Aneja, S., 2015. CONCOR: context-aware community-oriented routing for intermittently connected network. EURASIP Journal on Wireless Communications and Networking, 2015(1), p.148.

[8] Johari, R., Gupta, N. and Aneja, S., 2013, November. CACBR: context aware community based routing for intermittently connected network. In Proceedings of the 10th ACM symposium on Performance evaluation of wireless ad-hoc, sensor, \& ubiquitous networks (pp. 137-140). ACM.

[9] Johari, R., Gupta, N. and Aneja, S., 2013, January. DSG-PC: dynamic social grouping based routing for non-uniform buffer capacities in DTN supported with periodic carriers. In International Conference on Heterogeneous Networking for Quality, Reliability, Security and Robustness (pp. 1-15). Springer, Berlin, Heidelberg.

[10] Grasic, S., Davies, E., Lindgren, A. and Doria, A., 2011, September. The evolution of a DTN routing protocol-PRoPHETv2. In Proceedings of the 6th ACM workshop on Challenged networks (pp. 27-30). ACM.

[11] Boldrini, C., Conti, M., Delmastro, F. and Passarella, A., 2010. Context-and social-aware middleware for opportunistic networks. Journal of Network and Computer Applications, 33(5), pp.525-541.

[12] Vu, L., Do, Q. and Nahrstedt, K., 2010. Comfa: Exploiting regularity of people movement for message forwarding in community-based delay tolerant networks.

[13] Cabaniss, R., Madria, S., Rush, G., Trotta, A. and Vulli, S.S., 2010, December. Dynamic social grouping based routing in a mobile ad-hoc network. In 2010 International Conference on High Performance Computing (pp. 1-8). IEEE

[14] Hui, P., Crowcroft, J. and Yoneki, E., 2010. Bubble rap: Social-based forwarding in delay-tolerant networks. IEEE transactions on mobile computing, 10(11), pp.1576-1589.Wre

[15] Costa, P., Mascolo, C., Musolesi, M. and Picco, G.P., 2008. Socially-aware routing for publishsubscribe in delay-tolerant mobile ad hoc networks. IEEE Journal on selected areas in communications, 26(5), pp.748-760.

[16] Spyropoulos, T., Psounis, K. and Raghavendra, C.S., 2005, August. Spray and wait: an efficient routing scheme for intermittently connected mobile networks. In Proceedings of the 2005 ACM SIGCOMM workshop on Delay-tolerant networking (pp. 252-259). ACM.

[17] Lindgren, A., Doria, A. and Schelén, O., 2003. Probabilistic routing in intermittently connected networks. In ACM International Symposium on Mobilde Ad Hoc Networking and Computing, MobiHoc 2003: 01/06/2003-03/06/2003.

[18] Vahdat, A. and Becker, D., 2000. Epidemic routing for partially connected ad hoc networks.

[19] Watts, D.J. and Strogatz, S.H., 1998. Collective dynamics of 'small-world'networks. nature, 393(6684), p. 440 .

[20] Raj, V.S. and Chezian, R.M., 2013. DELAY-Disruption Tolerant Network (DTN), its Network Characteristics and Core Applications. International Journal of Computer Science and Mobile Computing, 2(9), pp.256-262. 
International Journal of Security and Its Applications Vol. 13, No. 4 (2019) 